\title{
Origin of monoclinic distortion and its impact on the electronic properties in $\mathrm{KO}_{2}$
}

\author{
Olga Sikora, ${ }^{1, *}$ Dorota Gotfryd,${ }^{2,3, \dagger}$ Andrzej Ptok, ${ }^{1, \dagger}$ Małgorzata Sternik, ${ }^{1}$ \\ Krzysztof Wohlfeld, ${ }^{2}$ Andrzej M. Oleś, ${ }^{3,4}$ and Przemysław Piekarz ${ }^{1, \S}$ \\ ${ }^{1}$ Institute of Nuclear Physics, Polish Academy of Sciences, W. E. Radzikowskiego 152, PL-31342 Kraków, Poland \\ ${ }^{2}$ Institute of Theoretical Physics, Faculty of Physics, University of Warsaw, Pasteura 5, PL-02093 Warsaw, Poland \\ ${ }^{3}$ Institute of Theoretical Physics, Jagiellonian University, Profesora Stanistawa Łojasiewicza 11, PL-30348 Kraków, Poland \\ ${ }^{4}$ Max Planck Institute for Solid State Research, Heisenbergstrasse 1, D-70569 Stuttgart, Germany
}

(Dated: July 31, 2020)

\begin{abstract}
We use the density functional theory and lattice dynamics calculations to investigate the properties of potassium superoxide $\mathrm{KO}_{2}$ in which spin, orbital, and lattice degrees of freedom are interrelated and determine the low-temperature phase. After calculating phonon dispersion relations in the high-temperature tetragonal $I 4 / \mathrm{mmm}$ structure, we identify a soft phonon mode leading to the monoclinic $C 2 / c$ symmetry and optimize the crystal geometry resulting from this mode. Thus we reveal a displacive character of the structural transition with the group-subgroup relation between the tetragonal and monoclinic phases. We compare the electronic structure of $\mathrm{KO}_{2}$ with antiferromagnetic spin order in the tetragonal and monoclinic phases. We emphasize that realistic treatment of the electronic structure requires including the local Coulomb interaction $U$ in the valence orbitals of the $\mathrm{O}_{2}^{-}$ions. The presence of the 'Hubbard' $U$ leads to the gap opening at the Fermi energy in the tetragonal structure without orbital order but with weak spin-orbit interaction. We remark that the gap opening in the tetragonal phase could also be obtained when the orbital order is initiated in the calculations with a realistic value of $U$. Finally, we show that the local Coulomb interactions and the finite lattice distortion, which together lead to the orbital order via the Jahn-Teller effect, are responsible for the enhanced insulating gap in the monoclinic structure.
\end{abstract}

\section{INTRODUCTION}

Despite of their simple stoichiometry, alkali $R_{2}$ superoxides [1] (with $R=\mathrm{Na}, \mathrm{K}, \mathrm{Rb}, \mathrm{Cs}$ ) are complex magnetic systems with couplings between spin, orbital and lattice degrees of freedom. They consist of alkali cations $R$ and the superoxide $\mathrm{O}_{2}^{-}$anions, i.e., charged oxygen molecules with an additional electron. The superoxide ion is interesting by itself and plays a role e.g. in biological processes [2]. In the crystal structure it provides partially filled $p$-electron bands that are responsible for a rich variety of phenomena.

Diffraction experiments [3-6] revealed multiple structural phase transitions that can be related to orientation of oxygen molecules. At high temperature the crystal structure is of cubic $\mathrm{NaCl}$ type with disoriented oxygen molecules, and upon decreasing temperature the symmetry in $\mathrm{KO}_{2}, \mathrm{RbO}_{2}$, and $\mathrm{CsO}_{2}$ is lowered to the bodycentered tetragonal $(I 4 / \mathrm{mmm})$ with average $\mathrm{O}_{2}$ orientation parallel to the tetragonal axis. With further cooling all three compounds transform to incommensurate phases and eventually reach low-temperature ordered structures with the onset of magnetic order [7].

Except for many similar properties, recent experimental and theoretical works show that even minor features distinguishing the alkali compounds, such as the extent of the oxygen molecule rotation from the tetragonal axis [8]

\footnotetext{
* corresponding author; e-mail: sikora@wolf.ifj.edu.pl

$\dagger$ e-mail: dorota.gotfryd@fuw.edu.pl

¥ e-mail: aptok@mmj.pl

$\S$ e-mail: piekarz@wolf.ifj.edu.pl
}

or the position of an alkali cation $p$-levels [9] can strongly influence their magnetic behavior. One of the reasons of this behavior is the competition between different exchange and superexchange mechanisms in systems which reveal significant geometrical and spin-orbital frustration [10-12].

In potassium dioxide $\mathrm{KO}_{2}$, the high temperature cubic structure [13] transforms at $395 \mathrm{~K}$ to the tetragonal $I 4 / \mathrm{mmm}$ phase $[5,6]$, presented in Fig. 1(a). Below $231 \mathrm{~K}, \mathrm{KO}_{2}$ is found in the incommensurate phase and at $196 \mathrm{~K}$ its crystal structure stabilizes in the monoclinic $C 2 / c$ phase depicted in Fig. 1(d). Further lowering of the symmetry leads to the triclinic phase, where a long-range $C$-type antiferromagnetic (AFM) order, with opposite spin orientations in two adjacent ferromagnetic (00l) oxygen layers was detected below the Néel temperature $T_{\mathrm{N}} \simeq 7 \mathrm{~K}$ by the neutron scattering [14]. In early theoretical studies, the occurrence of the AFM order was connected to the coherent tilting of $\mathrm{O}_{2}$ molecules observed in low-temperature phases $[4,15,16]$. The role of Jahn-Teller distortions at lower temperatures has been indicated [17] and the effect of the $\mathrm{O}_{2}$ tilting on the semiconducting propeties of $\mathrm{KO}_{2}$ was discussed within the $p$-electron correlated models [18].

The electronic band structure calculations for the tetragonal phase of potassium dioxide [10, 11], performed within the density functional theory (DFT), found the metallic ground state in disagreement with the experimental observations. This indicates the importance of electron correlations, and indeed, the combination of moderate $U$ and spin-orbit coupling (SOC) included within the GGA $+U$ approach opens an insulating gap of the order of $1.0 \mathrm{eV}[11,19]$. Larger values of the gap $(\sim 2$ - 
$5 \mathrm{eV}$ ) were obtained within the hybrid functional calculations and non-self-consistent many-body perturbation theory performed for the tetragonal structure with the tilted $\mathrm{O}_{2}^{-}$molecules [20].

The monoclinic structure of $\mathrm{KO}_{2}$ expected to be more stable at low temperature has been studied using ab initio methods and its formation energy compared to other potassium oxides [21-23]. The lattice distortion was found to be accompanied by two-sublattice orbital order [21]. In some other studies [11, 24], a simplified structure was considered with potassium ions in the tetragonal positions and all oxygen molecules tilted coherently, what implies the ferro-orbital order. Due to the lowering of symmetry and the onset of orbital order, the phase with lattice distortion reveals an insulating gap in the presence of the interaction $U$ alone $[11,24]$.

The studies presented until now focused mainly on the electronic properties of $\mathrm{KO}_{2}$. They uncover the cooperative scenario, in which the local Coulomb interactions $\propto U$ and the Jahn-Teller effect play an important role in the low-temperature phase [17]. However, a detailed mechanism of the structural transition, which breaks the tetragonal symmetry and leads to the monoclinic $C 2 / c$ phase has not been explained so far. In particular, the lattice dynamics in $\mathrm{KO}_{2}$ was never studied in the theory, and the role of phonons in the tetragonal-monoclinic transition is unknown.

Here we investigate the origin of the monoclinic phase and present a comprehensive description of its structural, dynamical, and electronic properties. We calculate phonon dispersion relations to demonstrate dynamical instability of the tetragonal cell at low temperatures, and use the lowest soft mode at the $N$ point of the Brillouin zone to generate and optimize the monoclinic $C 2 / c$ structure with rotated oxygen molecules and distorted potassium sublattice. We analyze its electronic properties and the origin of orbital order. The low-temperature magnetic order is also discussed. Special attention is given to possible mechanisms leading to the insulating behavior, i.e., the origin of the gap in the presence of either local Coulomb interaction $U$, or SOC, or both interactions acting jointly.

The paper is organized as follows. The calculation method is described in Sec. II. In Sec. III we discuss structural properties of the tetragonal and monoclinic phase of $\mathrm{KO}_{2}$, and analyze the phonon dispersion relations. We show the soft mode in the tetragonal structure that leads to the monoclinic distortion and demonstrate the stability of the monoclinic phase. Sec. IV B presents electronic band structures in both phases, while we discuss the source of magnetic order in Sec. IV C, and the origin of orbital order in the low temperature structure in Sec. IV D. Electronic densities of states are investigated in Sec. V. To uncover the mechanisms responsible for the gap opening, we consider models with either the Coulomb $U$, or with the SOC or both. In Sec. VI we conclude with a summary of the results and discussion of open questions. Appendix A presents calculations for the tetragonal cell in which an orbital order is initiated and stabilized by the Coulomb $U$. Finally, Appendix B shows the electronic densities of states with partial distortions of the tetragonal structure, to show separately the impact of the potassium and oxygen displacements on the electronic structure.

\section{COMPUTATIONAL DETAILS}

The spin-polarized DFT calculations were performed within the projector augmented-wave (PAW) method [25] using the Vienna Ab initio Simulation Package (VASP) [26-28]. The exchange-correlation potential was obtained by the generalized gradient approximation (GGA) in the form proposed by Perdew, Burke, and Enzerhof (PBE) [29]. Strong local electron interactions were included within the DFT $+U$ scheme [30], and we assume the intraorbital Coulomb parameter $U=4.0 \mathrm{eV}$ as in the earlier studies [9, 21], and Hund's exchange $J=0.62 \mathrm{eV}[10]$ on the oxygen $2 p$ orbitals. We also investigated the impact of the SOC [31] on the electronic structure.

We performed calculations for the AFM spin order found in the experiment [14], taking into account noncollinear spin configurations in calculations for the monoclinic phase. Optimization of both the structural parameters and the electronic structure in the tetragonal (monoclinic) phase were performed using a $16 \times 16 \times 8$ $(8 \times 16 \times 8)$ Monkhorst-Pack grid [33] of $\mathbf{k}$-points. The energy cut-off for the plane-wave expansion was equal to $520 \mathrm{eV}$. We checked the convergence of the structural parameters for the tetragonal phase by increasing its value to $700 \mathrm{eV}$ and found very small changes (by less than $0.3 \%$ ) of the lattice constants and the $\mathrm{O}-\mathrm{O}$ bond length. The structures were relaxed using the conjugate gradient technique with the energy convergence criteria set at $10^{-7}$ and $10^{-5} \mathrm{eV}$ for the electronic and ionic iterations, respectively. The electronic density of states (DOS) was calculated using the tetrahedron method.

For the relaxed structures, the phonon dispersion relations as well as the total and element-projected phonon DOS were calculated using the direct method [34] implemented in the PHONON software [35]. In this approach, the Hellmann-Feynman forces acting on all atoms in a given supercell are obtained by single-atom displacements from their equilibrium positions. We note that such atomic displacements do not change the insulating state of the system. The force-constant matrix elements are derived using the singular-value decomposition (SVD) technique and then the dynamical matrix is obtained. To describe the longitudinal optic-transverse optic (LO-TO) splitting induced by macroscopic polarization, the static dielectric tensor and Born effective charges were determined using density functional perturbation theory [36]. The phonon energies and polarization vectors are calculated by the exact diagonalization of the dynamical matrix. 
(a)

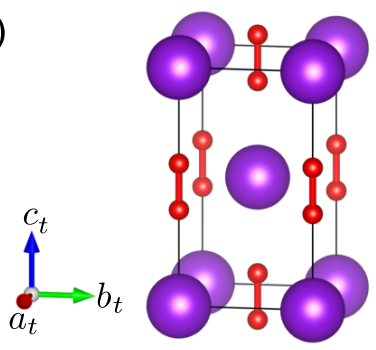

(d)

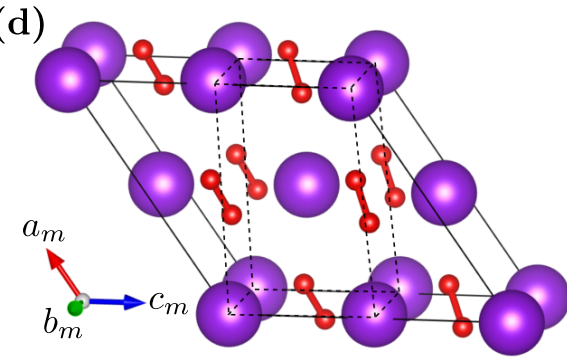

(b)

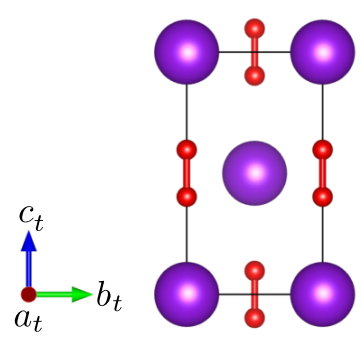

(e)

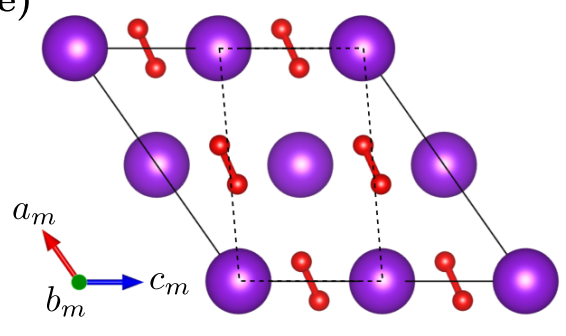

(c)

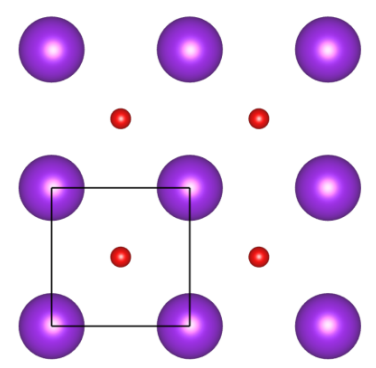

(f)

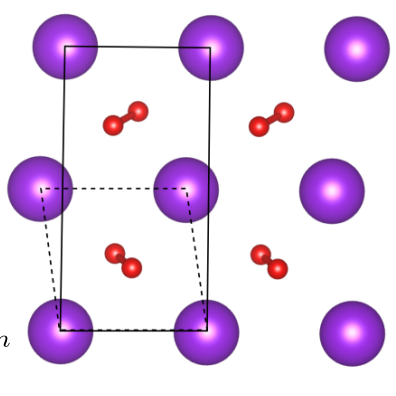

FIG. 1. Schematic view of the crystal structures of the potassium dioxide $\mathrm{KO}_{2}$. Left panels show: (a) high temperature $I 4 / \mathrm{mmm}$ tetragonal cell and (d) moderate temperature $C 2 / \mathrm{c}$ monoclinic cell, with oxygen molecules tilted from the vertical direction. $\mathrm{K}$ and $\mathrm{O}_{2}$ units are shown by violet balls and pairs of red balls. Panel (e) reveals the relation between the tetragonal cell shown along the $a_{t}$ direction in panel (b) with the monoclinic cell projected along the $b_{m}$ direction. Similarly, the relation between the bases of the cells for the tetragonal (c) and monoclinic (f) phase is shown for the planes $a_{t} b_{t}$ and $b_{m} c_{m}$, respectively. The image was rendered using VESTA software [32].

\section{CRYSTAL STRUCTURES AND THEIR STABILITY}

\section{A. Tetragonal phase}

By relaxing the tetragonal cell with two $\mathrm{KO}_{2}$ formula units, see Fig. 1(a), we obtained the lattice constants $a_{t}=3.979 \AA$ and $c_{t}=6.928 \AA$, both in a good agreement with the experimental data, $a_{t}=4.034 \AA$ and $c_{t}=6.699 \AA[5,6]$. In this structure, oxygen molecules are oriented along the $c_{t}$ direction. The calculated $\mathrm{O}-\mathrm{O}$ bond length of $1.361 \AA$ is larger than the experimental values of $1.28(7) \AA[5]$ and $1.28(2) \AA[6]$ typically presented in the literature. In fact, these values are underestimated because the librational motions of $\mathrm{O}_{2}$ molecule are not considered in structural models used to evaluate the diffraction data $[37,38]$. Reinterpretation of the same data with a model allowing the motion of the $\mathrm{O}_{2}$ molecule around the $c$ axis leads to a bond length in the range of $1.32-1.35 \AA$ [17]. Similar values, ranging between 1.31 and $1.34 \AA$, are found in the study performed using the near edge $\mathrm{X}$-ray absorption fine structure experiment and multiple scattering calculations on $\mathrm{KO}_{2}$ [39], and in other studies on various superoxides [38, 40].

The phonon dispersion relations calculated for the tetragonal (insulating) structure are presented in Fig. 2(a), along the high symmetry directions of the first Brillouin zone (FBZ) shown in the inset, and the phonon
DOS is displayed in Fig. 2(b). The contributions of oxygen and potassium atoms to individual phonon branches and DOS are depicted in blue and red, respectively. The entire phonon spectrum of $\mathrm{KO}_{2}$ consists of two separated energy ranges. The low-energy part below $30 \mathrm{meV}$ includes the acoustic and optic vibrations of $\mathrm{K}$ and $\mathrm{O}$ atoms. In contrast, the high-energy dispersion branch around $150 \mathrm{meV}$ corresponds to the internal vibrations in the individual oxygen molecules. Two phonon branches exhibit imaginary frequencies (plotted with negative values) along some directions of the reciprocal space, reflecting dynamical instability of the tetragonal structure at low temperatures. These imaginary modes arise predominantly due to the vibrations of $\mathrm{K}$ atoms, however, small contributions of the oxygen movements are also visible in the partial phonon DOS presented in Fig. 2(b).

At the $\Gamma$ point, the group theory predicts the following decomposition of the irreducible representations of the phonon modes: $\Gamma=A_{1 g}+2 A_{2 u}+E_{g}^{(2)}+2 E_{u}^{(2)}$, where $A_{1 g}$ and $E_{g}$ are Raman-active modes, while $A_{2 u}$ and $E_{u}$ are infrared-active modes. In agreement with the dimension of irreducible representations, all $E$ modes are doubly degenerate at the FBZ center. The energies of the TO modes at the $\Gamma$ point are collected in Tab. I. Two infrared optical modes exhibit the LO-TO splitting and the LO mode is shifted to higher energies due to the long-range electric polarization. The Raman measurements of $\mathrm{KO}_{2}$ performed at $300 \mathrm{~K}$ confirm the presence of the strong scattering at $141.48 \mathrm{meV}$ [41], which is attributed to the 
(a) tetragonal $(\mathrm{I} 4 / \mathrm{mmm})$

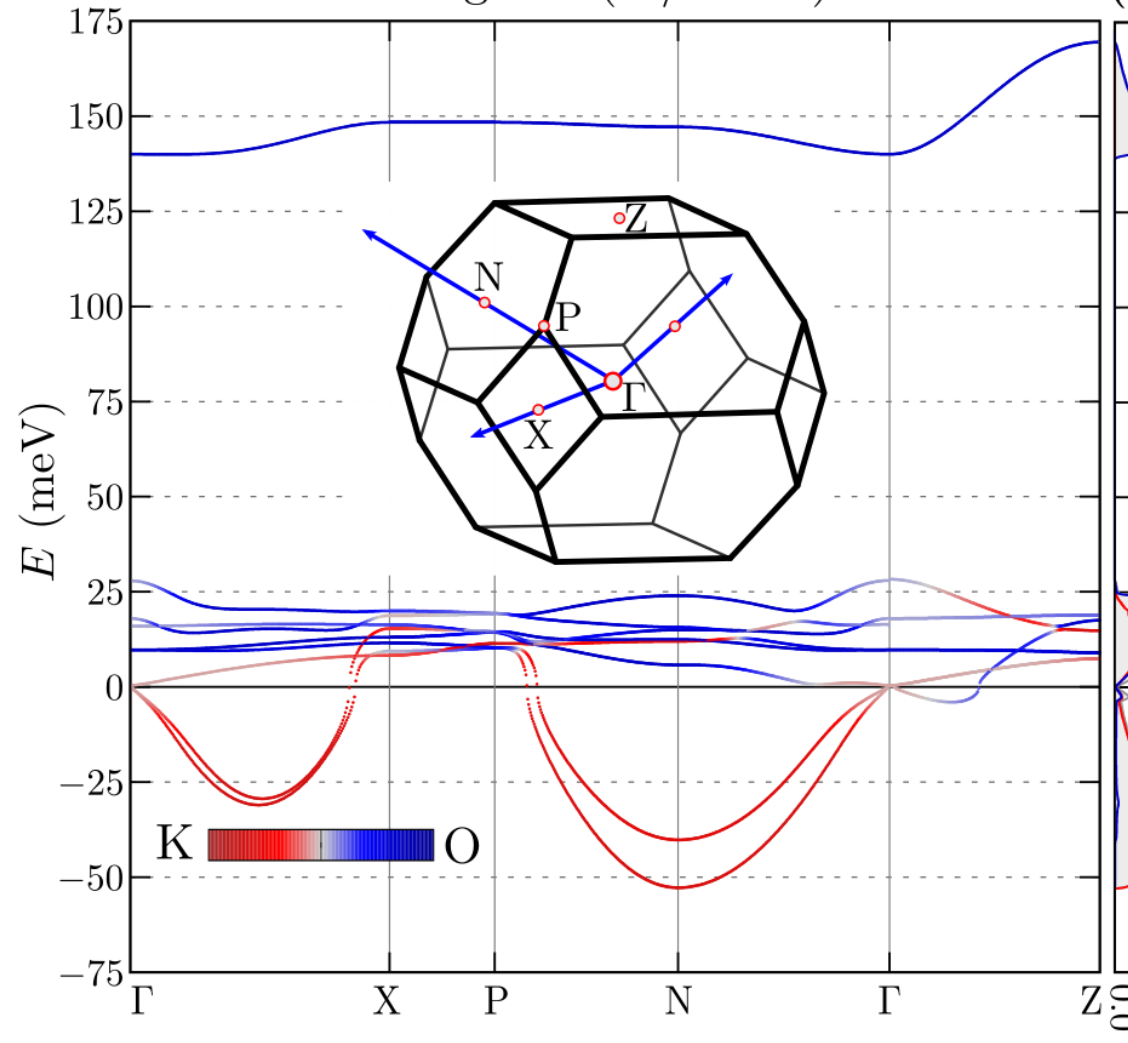

(c)

monoclinic $(\mathrm{C} 2 / \mathrm{c})$

(b)

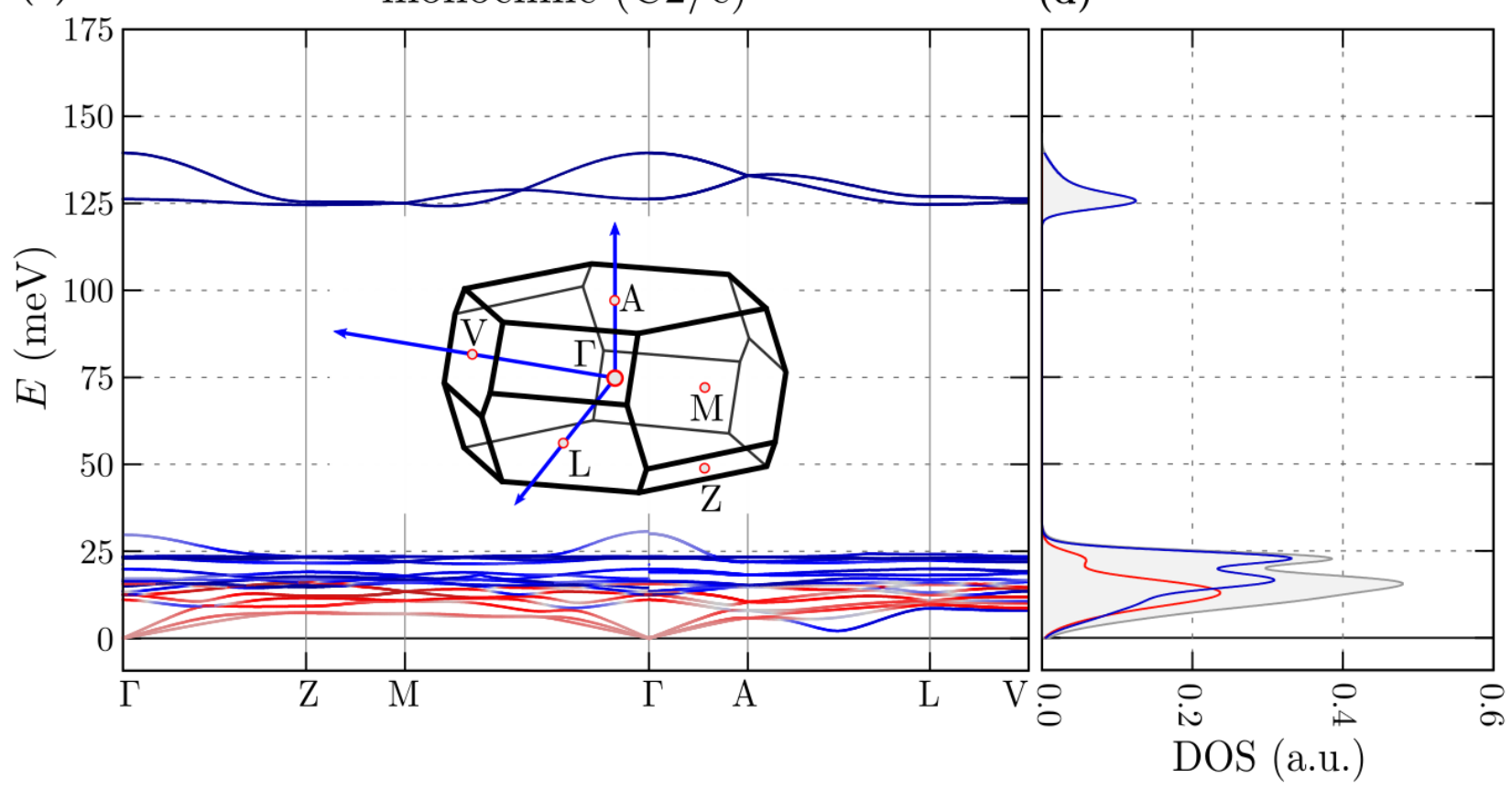

FIG. 2. Phonon dispersion curves along high symmetry directions [cf. the first Brillouin zone (FBZ) in the insets] for: (a) the tetragonal $I 4 / \mathrm{mmm}$ and (c) monoclinic $C 2 / \mathrm{c}$ phase of $\mathrm{KO}_{2}$. The color of lines indicates the contribution of $\mathrm{K}$ or $\mathrm{O}$ atoms (cf. color bar). In the right panels, the phonon density of states (DOS) is shown for (b) tetragonal and (d) monoclinic phase, with gray, red and blue corresponding to the total, partial $\mathrm{K}$ and partial O phonon DOS, respectively. The lattice dynamics calculations of the tetragonal structure (a) reveal several soft modes with imaginary frequency (presented as negative values), predominantly associated with $\mathrm{K}$ atoms. The lowest mode at the $\mathrm{N}$ point leads to the monoclinic symmetry $(C 2 / c)$. Phonon dispersion relations for the optimized $C 2 / c$ structure (c) do not reveal the soft modes, confirming the stability of the monoclinic phase. 
TABLE I. Phonon modes with their irreducible representations (IR), energies, and activities (R-Raman and I-infrared), as obtained from the phonon calculations at the $\Gamma$ point in the tetragonal and monoclinic phases of the potassium dioxide $\mathrm{KO}_{2}$. For the infrared phonons only the TO modes are indicated. In parentheses the measured energies of Raman modes [41] are presented (when available).

\begin{tabular}{ccc|cccc}
\hline \hline \multicolumn{3}{c}{ Tetragonal $I 4 / m m m$} & \multicolumn{4}{c}{ Monoclinic $C 2 / c$} \\
$\mathrm{IR}$ & $E(\mathrm{meV})$ & Activity & $\mathrm{IR}$ & $E(\mathrm{meV})$ & Activity \\
\hline$E_{g}$ & 9.73 & $\mathrm{R}$ & $B_{g}$ & 11.09 & $(8.06)$ & $\mathrm{R}$ \\
$A_{2 u}$ & 15.98 & $\mathrm{I}$ & $A_{u}$ & 12.55 & & $\mathrm{I}$ \\
$E_{u}$ & 18.00 & $\mathrm{I}$ & $A_{g}$ & 12.59 & $(10.04)$ & $\mathrm{R}$ \\
$A_{1 g}$ & $139.99(141.48)$ & $\mathrm{R}$ & $B_{g}$ & 13.35 & $(11.04)$ & $\mathrm{R}$ \\
& & & $B_{u}$ & 13.51 & & $\mathrm{I}$ \\
& & $B_{g}$ & 15.65 & $(15.13)$ & $\mathrm{R}$ \\
& & $A_{g}$ & 16.38 & $(17.36)$ & $\mathrm{R}$ \\
& & $B_{u}$ & 17.01 & & $\mathrm{I}$ \\
& & $A_{u}$ & 19.91 & & $\mathrm{I}$ \\
& & $B_{u}$ & 21.21 & & $\mathrm{I}$ \\
& & $A_{g}$ & 23.00 & $(25.42)$ & $\mathrm{R}$ \\
& & $B_{g}$ & 23.46 & $(26.16)$ & $\mathrm{R}$ \\
& & $A_{u}$ & 23.47 & & $\mathrm{I}$ \\
& & $B_{g}$ & 126.21 & & $\mathrm{R}$ \\
& & $A_{g}$ & 139.47 & $(141.98)$ & $\mathrm{R}$ \\
\hline \hline
\end{tabular}

stretching mode of $\mathrm{O}_{2}^{-}$ions.

After examining the crystal structure resulting from atomic displacements induced by the polarization vector of the lowest soft mode at the $\mathrm{N}$ point, we found the $C 2 / c$ monoclinic symmetry. This soft mode rotates oxygen molecules and induces shifts of potassium atoms along the $b_{t}$ direction. It shows that the tetragonal-tomonoclinic transition has a displacive character with the group-subgroup relation between the high-temperature and low-temperature symmetries. The group theory analysis indicates a few possible intermediate symmetries (subgroups of $I 4 / \mathrm{mmm}$ and supergroups of $C 2 / \mathrm{c}$ ), which may explain the existence of the incommensurate phase observed by diffraction studies [3].

\section{B. Monoclinic phase}

We have obtained the monoclinic $C 2 / c$ structure using the soft mode revealed by the calculations for the tetragonal structure, as discussed above. The monoclinic structure has a lower total energy with respect to the tetragonal phase, approximately $0.129 \mathrm{eV}$ per one formula unit (f.u.). For both phases, the total energies were calculated for the insulating AFM states. Within the optimization procedure, we found the lattice parameters, $a_{m}=8.098 \AA, b_{m}=4.200 \AA, c_{m}=8.178 \AA$, and $\beta=125.093^{\circ}$, similar to those obtained from the $\mathrm{x}$ ray diffraction: $a_{m}=7.880 \AA, b_{m}=4.036 \AA, c_{m}=$
$7.968 \AA$, and $\beta=122.85^{\circ}$ [3]. The calculated $\mathrm{O}-\mathrm{O}$ bond length is $1.359 \AA$, only slightly smaller than in the tetragonal phase. In the monoclinic cell two nonequivalent atoms $\mathrm{K}$ and $\mathrm{O}$ are placed at the Wyckoff positions $4 e(0,0.078,0.25)$ and $8 f(0.416,0,072,-0.017)$, respectively. We note that in contrary to the analysis of the experimental data [3] and some theoretical studies $[11,24]$, we find pronounced changes in the potassium positions with respect to the tetragonal structure. The relation between both crystal structures is shown in Fig. 1. The lattice parameters in the tetragonal and monoclinic phases are connected by the following approximate relations: $a_{m} \sim \sqrt{a_{t}^{2}+c_{t}^{2}}, b_{m} \sim b_{t}$, and $c_{m} \sim 2 a_{t}$ [cf. Figs. 1(a) and 1(d)]. The number of atoms in the monoclinic unit cell is twice as large as in the tetragonal one.

The lattice deformation induced by the structural transformation is best visible in the projections presented in Fig. 1. The relation between positions of $\mathrm{K}$ atoms in the bases of the tetragonal and monoclinic cell is presented in Fig. 1(f). The shifts of $\mathrm{K}$ atoms are harmonized with the rotations of $\mathrm{O}_{2}^{-}$anions. These rotations can be described as the coherent tilting $\left(24.4^{\circ}\right)$ in the $a_{m} c_{m}$ plane shown in Fig. 1(e), combined with rotations alternating along the $c_{m}$ direction [cf. Fig. 1(f)]. The total tilting of the molecule from the tetragonal $c_{t}$ direction amounts to $35.3^{\circ}$. Similar deformation was obtained by the relaxation of the $\mathrm{KO}_{2}$ structure without the symmetry constraints in Ref. [21]. The formation energy of $\mathrm{KO}_{2}$ was calculated for the $C 2 / c$ symmetry, however, a different setting of the lattice parameters for the distorted structure was chosen $[22,23]$. We note that a similar $C 2 / c$ structure was predicted in $\mathrm{SrO}_{2}$ from firstprinciples swarm structure searching simulations [42].

The monoclinic $C 2 / c$ structure is dynamically stable as shown in Fig. 2 (lower panels). Twice as many atoms in the primitive unit cell generate the doubled number of phonon branches in the monoclinic structure compared

\section{superoxide $\mathrm{O}_{2}^{-}$}

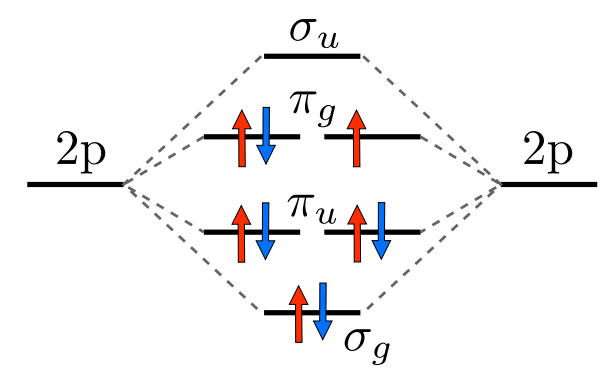

FIG. 3. Electronic energy levels for an isolated $\mathrm{O}_{2}$ molecule formed by the oxygen $2 p$ orbitals of both oxygen atoms, and their occupancy in the superoxide anion $\mathrm{O}_{2}^{-}$filled by nine electrons. 


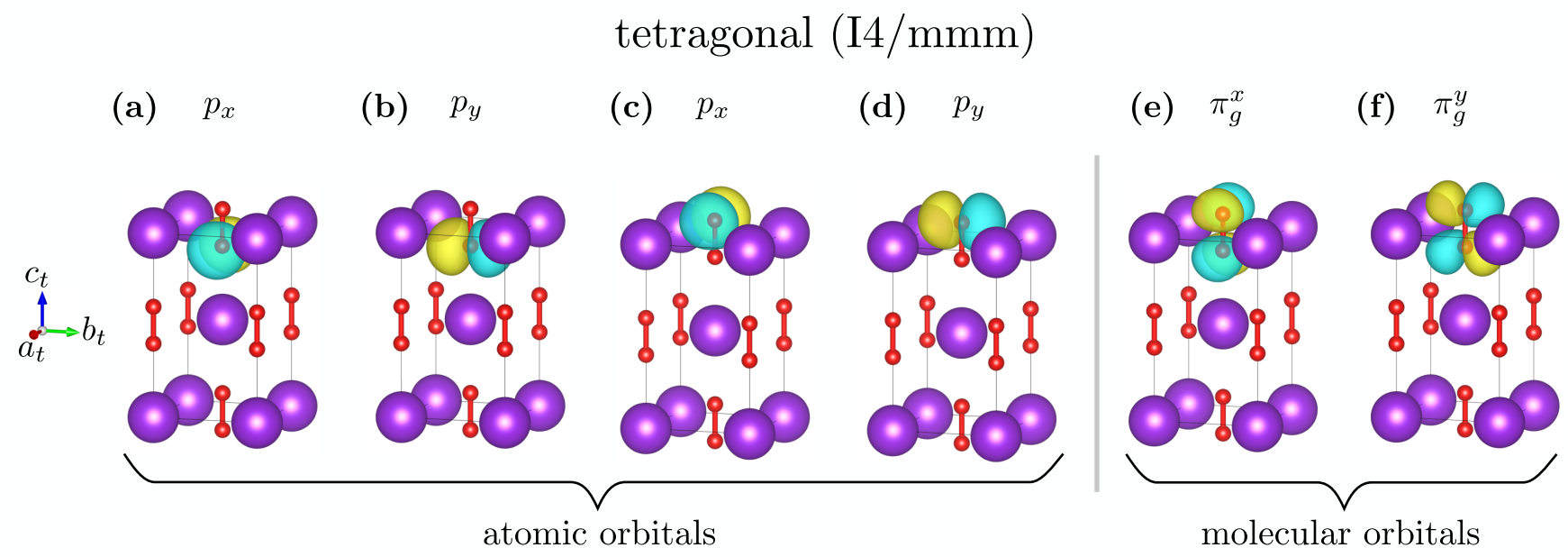

FIG. 4. Four atomic and two molecular valence orbitals in the tetragonal phase: (a)-(d) $p_{x}$ and $p_{y}$ atomic orbitals of the oxygen atoms, (e)-(f) $\pi_{g}^{x}$ and $\pi_{g}^{y}$ molecular orbitals of an $\mathrm{O}_{2}^{-}$molecule. The presented orbitals were obtained from the numerical analyzes of the DFT bands around the Fermi level. The image was rendered using vesTA software [32].

to tetragonal one, and because of low symmetry all degenerate modes are split. For both structures the upper limit of the low-energy part is very similar, but in the monoclinic phase two highest dispersion branches are shifted to lower energies. All phonons at the $\Gamma$ point are described by one-dimensional irreducible representations: $\Gamma=4 A_{g}+4 A_{u}+5 B_{g}+5 B_{u}$, where $A_{g}, B_{g}$ are Raman-active modes and $A_{u}, B_{u}$ are infrared-active modes. Their energies are presented in Tab. I. For the Raman modes, we obtained a good agreement with the experimental values measured at $80 \mathrm{~K}$ [41].

\section{ELECTRONIC PROPERTIES}

\section{A. Molecular orbitals for an $\mathrm{O}_{2}^{-}$molecule}

The $\mathrm{KO}_{2}$ crystal is ionic and thus its electronic properties are strongly associated with the orbital structure of the $\mathrm{O}_{2}^{-}$ions, in which the hybridization between atomic $2 p$ orbitals gives rise to bonding $\left\{\sigma_{g}, \pi_{u}\right\}$ and antibonding $\left\{\pi_{g}, \sigma_{u}\right\}$ molecular orbitals, see Fig. 3. An isolated $\mathrm{O}_{2}$ molecule with eight $2 p$ electrons has two unpaired electrons in the doubly degenerate $\pi_{g}$ level and a triplet ( $S=1)$ ground state, as required by the first Hund's rule. $\mathrm{KO}_{2}$ however includes $\mathrm{O}_{2}^{-}$anions with one extra electron coming from the alkali ion and the molecular configuration is [18] $\sigma_{g}^{2} \pi_{u}^{4} \pi_{g}^{3}$, as shown in Fig. 3 .

Important differences between the tetragonal and monoclinic phases, that may influence their electronic properties, are already visible when the molecular orbitals of the $\mathrm{O}_{2}^{-}$ion are considered either in the tetragonal or monoclinic crystal lattice. In the tetragonal phase, the molecular $\pi_{g}$ orbitals [Fig. 4(e)-(f)], located around the Fermi level, are combinations of either two $p_{x}$ or two $p_{y}$ atomic orbitals [cf. Fig. 4(a)-(d)]. We note that different combinations of these atomic orbitals lead to the fully occupied $\pi_{u}$ states, and the occupied bonding $\sigma$ orbital is composed of two $p_{z}$ orbitals (not shown). Transition from the tetragonal to monoclinic phase is associated with tilting of the $\mathrm{O}_{2}^{-}$ions, and this also leads to rotation of the molecular orbitals in space, as shown for the $\pi_{g}$-type orbitals in Fig. 5. Because of two inequivalent rotations of the oxygen ions, two different sets of orbitals are needed as bases for the valence $\pi_{g}$ states: $\{\alpha, \beta\}$ on one sublat-

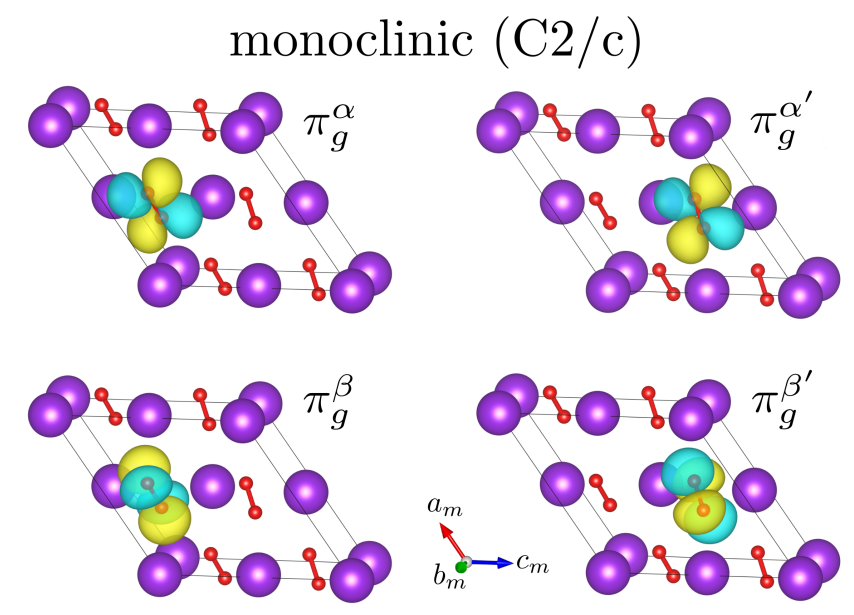

FIG. 5. Two inequivalent pairs of molecular valence orbitals in the monoclinic phase: $\left\{\pi_{g}^{\alpha}, \pi_{g}^{\beta}\right\}$ and $\left\{\pi_{g}^{\alpha^{\prime}}, \pi_{g}^{\beta^{\prime}}\right\}$, corresponding to two orientations of the oxygen molecule. The presented orbitals were obtained from the numerical analysis of the DFT bands around the Fermi level. The image was rendered using VESTA software [32]. 


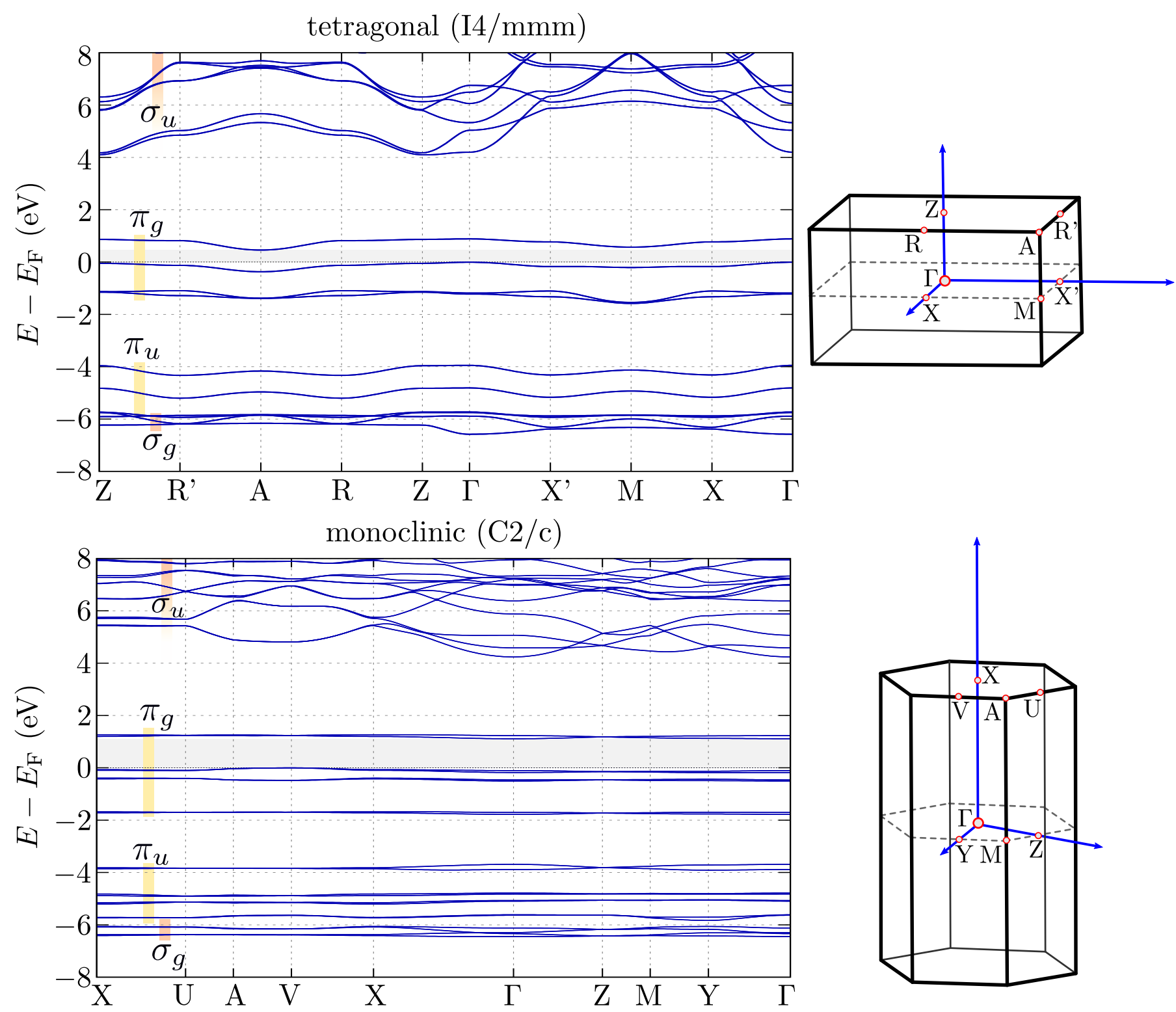

FIG. 6. Electronic band structure for the tetragonal (top panels) and monoclinic (bottom panels) phases in the magnetic unit cell. Right panels present the first (magnetic) Brillouin zones. Results for GGA+U+SOC calculations in the presence of the $A$-AFM order (cf. Fig. 7).

tice and $\left\{\alpha^{\prime}, \beta^{\prime}\right\}$ on the other.

\section{B. Electronic band structure}

The electronic band structure (Fig. 6) shows a few characteristic features that do not depend on the crystal geometry and the calculation scheme (i.e., including spin-orbit coupling or local Coulomb interaction). The $\sigma_{g}$ bands are located around $-6 \mathrm{eV}$ below the Fermi level. Slightly higher the bands formed by the bonding $\pi_{u}$ molecular orbitals are located, while a group of the antibonding $\pi_{g}$ bands is situated around the Fermi level.
In all these bands the role of the potassium orbitals is negligible [21]. The antibonding $\sigma_{u}$ orbitals are strongly intermixed with empty $\mathrm{K}$ states above $4 \mathrm{eV}$.

In the tetragonal phase, the splitting of the $\pi_{g}$ bands at the $\Gamma$ point is equal to approximately $25 \mathrm{meV}$, what is similar to the value reported previously in Ref. [10]. Contrary to dioxides of $4 d$ or $5 d$ elements, i.e., $\mathrm{RuO}_{2}$ [43] or $\mathrm{IrO}_{2}$ [44-47], the value of the SOC is relatively small in $\mathrm{KO}_{2}$. In fact, the $\mathrm{SOC}$ value strongly depends on the mass of compound components and plays an important role for massive atoms [48, 49], while here both atoms forming the structure are relatively light. In the monoclinic phase, the value of SOC can decrease by tilting of 
the $\mathrm{O}_{2}$ molecules [10].

The most apparent difference between the tetragonal and monoclinic electronic band structure is the increase of the charge gap from $0.5 \mathrm{eV}$ in the tetragonal phase to $1.1 \mathrm{eV}$ in the monoclinic phase, which is in a relatively good agreement with the activation energy $1.3 \mathrm{eV}$ obtained from the electrical conductivity measurements [18]. Also from x-ray absorption spectroscopy (XAS) measurements, a similar value of the gap was found [19]. This increase can be associated with the onset of the orbital order stabilized by the lattice distortion (discussed in more detail in Sec. V and the Appendices). Due to the folding of the Brillouin zone, we observe that the band degeneracy doubles. Additionally, we observe "flattening" of the bands in the monoclinic phase in relation to the tetragonal phase. It corresponds well with the experimental results, which indicate that the main effect of the structural phase transition on the electronic structure is the band narrowing [18].

\section{Magnetic order}

All results presented in this work are obtained for the $A$-type AFM $(A$-AFM) order [50], see Fig. 7. The investigated spin configuration for the tetragonal cell is presented in Fig. 7(a), with spins parallel to the oxygen molecule (we found the lowest energy for this spin configuration, although the values obtained for other orientations are very close). In the monoclinic phase, we assume spin directions to follow the rotation of molecules, therefore, the spin configuration is non-collinear as shown in Fig. 7(b). Nevertheless, the projections of the magnetic moments on the $c_{t}$ axis maintain the $A$-AFM order.

Earlier electronic structure calculations within the GGA $+U$ method showed lower energy of the ferromagnetic (FM) order with respect to the AFM state for small values of $U$ at oxygen ions, and the decreasing energy difference between these phases with growing $U$ [21]. With the increasing value of $U$, the character of electronic (a)

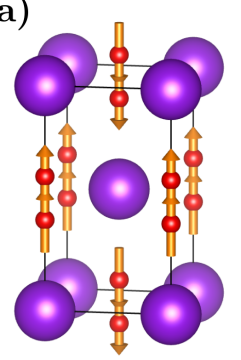

(b)

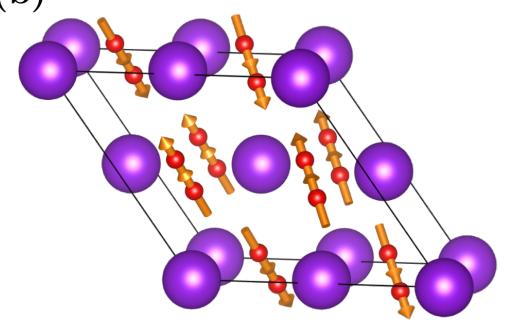

FIG. 7. The schematic view of the assumed $A$-AFM order in $\mathrm{KO}_{2}$ : (a) tetragonal and (b) monoclinic phase. Consecutive FM planes along the $c_{t}$ axis have alternating magnetic moments on $\mathrm{O}_{2}^{-}$molecules; conventions are the same as in Fig. 1. The image was rendered using VESTA software [32]. states changes from itinerant, which prefers the FM order, to localized, in which the FM and AFM interactions compete due to the anisotropic kinetic exchange between the $\mathrm{O}_{2}^{-}$anions [11]. The studies performed for a realistic spin-orbital model [12] demonstrated that the geometric frustration of magnetic interactions can be lifted and the $A$-AFM state is stabilized due to the orbital ordering.

Our calculations for the relaxed $C 2 / c$ structure show indeed very small energy differences between the FM and AFM configurations with slightly lower energy for the FM state regardless of the value of $U$. The negligibly small value $0.375 \mathrm{meV} /$ f.u. for $U=4 \mathrm{eV}$ confirms that magnetic interactions in $\mathrm{KO}_{2}$ are highly frustrated [12] Including additional effects like the superexchange and spin quantum fluctuations, not captured properly in the $\mathrm{DFT}+U$ methods, may lower the energy of the $A$-AFM state.

\section{Orbital order}

Calculations for the tetragonal cell performed with full symmetry do not break the degeneracy between the $\pi_{g}^{x}$ and $\pi_{g}^{y}$ orbitals. Their equal occupation can be demonstrated by plotting isosurfaces of the electronic density [see Fig. 8(a)] for the highest in energy, empty $\pi_{g}$ band (i.e., the hole density) in the $a_{t} b_{t}$ plane. Such calculations are analogous to restricted Hartree-Fock approach, while it is well known that a minimal energy is obtained only when the symmetry is broken in the orbital space and one looks for an unrestricted configuration with orbital order [51]. An orbitally ordered state with breaking of the $\pi_{g}^{x} / \pi_{g}^{y}$ symmetry can only be obtained if different orbital occupations are initiated at the beginning of optimization [52], as described in Appendix A. It should be also noted that the dynamical mean-field theory (DMFT) calculations performed for the paramagnetic Mott insu-

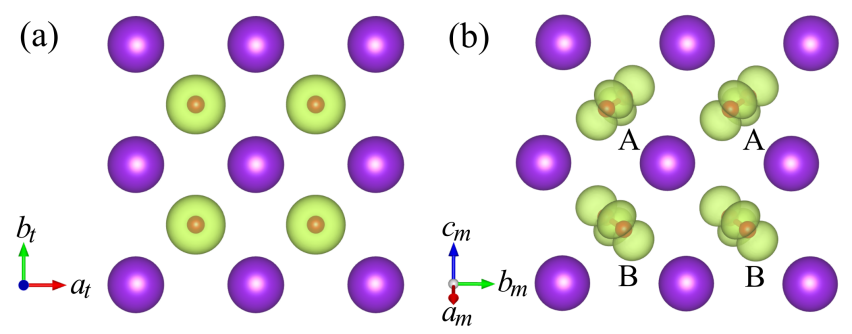

FIG. 8. Calculated isosurfaces of the hole density (in green) for the: (a) tetragonal and (b) monoclinic phase. In the tetragonal phase, due to the degeneracy of the oxygen ion orbitals $\pi_{g}^{x}$ and $\pi_{g}^{y}$, the density distribution is symmetric around the molecule axis. In the monoclinic phase the orbital order emerges as a result of the lattice distortion. The unoccupied orbitals are linear combinations of the molecular orbitals $\left\{\pi_{g}^{\alpha^{\prime}}, \pi_{g}^{\beta^{\prime}}\right\}$ and $\left\{\pi_{g}^{\alpha}, \pi_{g}^{\beta}\right\}$ on the sublattice A and B, respectively (cf. Fig. 5). The image was rendered using VESTA software [32]. 

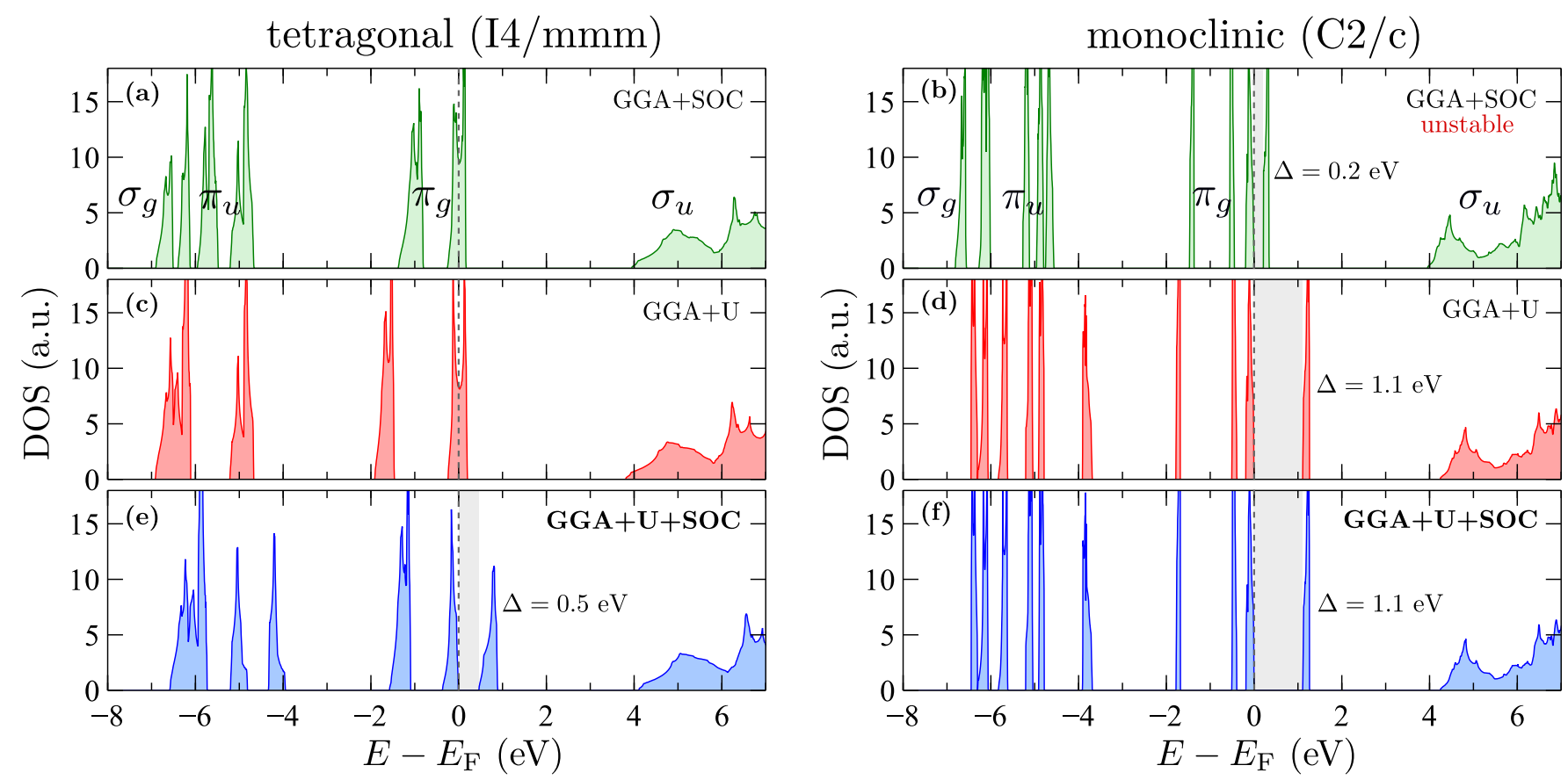

FIG. 9. Comparison of the electronic density of states (DOS) obtained for the tetragonal and the monoclinic structures (left and right panels, respectively) within GGA+SOC, GGA $+U$, and GGA $+U+\mathrm{SOC}$ calculations (panels from top to bottom). The assignment of each band to a particular $\mathrm{O}_{2}$ molecular orbital character is indicated in the top panels. The insulating gap $\Delta$ is defined as the difference between the energies of the last occupied and first unoccupied states.

lating state revealed the orbital fluctuations at high temperatures and the orbital order emerging only in the lowtemperature (distorted) phase [24]. The results obtained for $\mathrm{KO}_{2}$ can be compared with the DFT+DMFT studies for $\mathrm{RbO}_{2}$, which show the paramagnetic Mott insulating state at room temperature, obtained without including dynamic distortions of the tetragonal structure, and indicate a complex spin-orbital order at low temperatures [53].

Lattice distortions present in the monoclinic cell lower the symmetry of crystal field surrounding the superoxide anion and the degeneracy between the $\pi_{g}$ orbitals is now broken. Indeed, we find a two-sublattice orbital order in the density plot shown in Fig. 8(c) for the $b_{m} c_{m}$ plane, with alternating orbital states along the $c_{m}$ axis. The density distribution reflects the orbital states in the electrostatic fields of $\mathrm{K}^{+}$ions after distortion, in the bases $\left\{\alpha^{\prime}, \beta^{\prime}\right\}$ (sublattice A) and $\{\alpha, \beta\}$ (sublattice B), in analogy to the occupied linear combinations of $\left\{t_{2 g}\right\}$ orbitals in the vanadium perovskites with defects [51].

We obtain the same orbital order regardless whether the Coulomb $U$ is included (we performed calculations for $U=0$ for the unrelaxed structure). These results suggest the dominating role of monoclinic distortion in determining the orbital state. The exchange effects are too small to influence the orbital pattern, and we can also expect that they are highly frustrated.

A similar two-sublattice orbital order was found in an earlier study for $\mathrm{KO}_{2}$ [21]. Interestingly, within another approach [11, 24], potassium ions remained in their tetragonal positions and coherent tilting of oxygen molecules with accompanying ferro-orbital order was considered.

\section{ORIGIN OF THE INSULATING GAP}

At first sight, the origin of the insulating gap which opens at the Fermi level seems to be qualitatively similar in the tetragonal and monoclinic phases. Firstly, in both cases the insulating gap sets inside the bands exhibiting the $\pi_{g}$ character. Secondly, the gap size is "just" around twice larger in the monoclinic than in the tetragonal phase - which can be explained by invoking the shifts of the potassium ions and tilting of the $\mathrm{O}_{2}$ molecules in the monoclinic phase (cf. Appendix. B). Indeed, a more detailed study of the origin of the insulating gap presented below confirms the similarities between the two cases - albeit with one, rather important, difference.

\section{A. Tetragonal phase}

Let us start with the tetragonal phase and investigate how the electronic DOS depends on two crucial on-site interactions present in the system: the spin-orbit coupling and the Hubbard-like Coulomb repulsion $U$. To this end, we calculate the DOS using GGA+SOC, $\mathrm{GGA}+U$, as 
well as GGA $+U+$ SOC approaches, cf. Fig. 9 left panels from top to bottom, respectively. We note in passing that the unshown "pure" GGA result cannot be distinguished from the GGA+SOC case [Fig. 9(a)]. Whereas the assignment of the band character to particular molecular orbitals does not change upon including the on-site terms in the calculations, the insulating gap only opens in the GGA $+U+$ SOC case [Fig. 9(e)], i.e., when both types of the on-site interactions are included. The explanation of this phenomenon is three-fold:

First, we note that when both interactions are excluded, as in the GGA approach, the $\pi_{g}$ orbitals around the Fermi levels are degenerate (see Sec. IV D). Second, even when the spin-orbit coupling is included, its value is too small to open the gap (the band width is much bigger than the band splitting induced by the SOC). Third, on the mean-field level that is captured by the $\mathrm{DFT}+U$ calculations, the Hubbard $U$ can only induce the insulating gap when the existence of the non-degenerate $\pi_{g}$ bands is allowed by the spin-orbit coupling [Fig. 9(e)]. We stress that the latter leads to the distinct energies of the $\pi_{g}^{x} \pm i \pi_{g}^{y}$ orbitals [10], i.e., orbitals whose charge densities are exactly the same.

There is however one caveat: As already mentioned above, in calculations with the tetragonal symmetry, breaking of the orbital degeneracy of the $\pi_{g}$ bands with distinct charge densities is not allowed. The orbital order can be nevertheless "artificially" initiated, leading to a stable state and the opening of the gap of $0.65 \mathrm{eV}$ (see Appendix A).

\section{B. Monoclinic phase}

Let us now move to the monoclinic structure, and following the same sequence of calculation schemes, examine the impact of monoclinic distortion on the electronic DOS, see Fig. 9 (right panels). In contrast to the tetragonal case, without $U$ we are unable to stabilize the structure shown in Fig. 7(b) [54], it is interesting though to calculate the DOS with $U=0$ for the unrelaxed cell (i.e., the structure optimized with $U=4 \mathrm{eV}$ ). Both in the "pure" GGA (unshown) and in the GGA+SOC approach [Fig. 9(b)], we observe a small gap of $0.2 \mathrm{eV}$ (the splitting is however much larger than the energy scale of the SOC). This result, together with the orbital order reported in Sec. IV D, is an indication of the presence of the Jahn-Teller effect in $\mathrm{KO}_{2}$, in agreement with Nandy et al. [21].

The fact that the monoclinic crystal structure leads to the splitting of the two $\pi_{g}$ orbitals around the Fermi level means that the system is already "prepared" for the $U$-induced gap and the SOC which mixes the orbital states is not required. Indeed, when the Hubbard $U$ is included [Fig. 9(d)] the gap of $1.1 \mathrm{eV}$ is obtained, and in contrast to the tetragonal case, the impact of the SOC is negligible [cf. the results obtained from $\mathrm{GGA}+U+\mathrm{SOC}$ calculations of Fig. 9(f)].

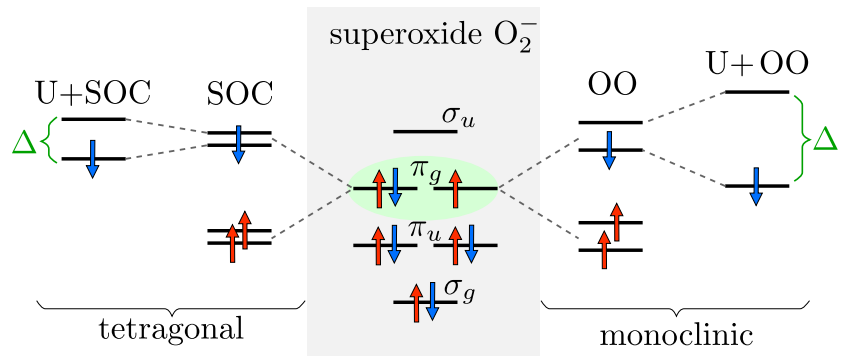

FIG. 10. Schematic view of the mechanism contributing to the opening of insulating (Mott-Hubbard) gap in $\mathrm{KO}_{2}$ by splitting degenerate $\pi_{g}$ orbitals located at the Fermi level in the tetragonal (left) and monoclinic (right) phase. In both structures the energy levels of spins up and down are separated by spin polarization gap. The spin-orbit coupling (SOC) leads to lifting of the orbital degeneracy in the tetragonal phase. The intrinsic orbital order (OO) induced by the JahnTeller effect plays a similar role in the monoclinic structure. In both phases, finite interaction $U$ plays a crucial role and enhances the insulating gap to its finite value $\Delta$.

The value of the insulating gap, more than twice as large as in the tetragonal case, is another remarkable difference between the two phases. Whereas this phenomenon was discussed in a quantitative manner in Sec. IV B, it can now be naturally explained by invoking that the insulating gap should not only incorporate the Hubbard $U$-induced gap but also the 'Jahn-Teller gap', as indicated by the above-discussed GGA (or GGA+SOC) electronic band structure. The simplified model of the monoclinic distortion, discussed in Appendix B with $U=4 \mathrm{eV}$, reveals that even a small deviation from the tetragonal symmetry (just enough to lift the orbital degeneracy) leads to a predominantly $U$-induced gap of about $0.8 \mathrm{eV}$. We can thus roughly estimate that the full monoclinic distortion contributes about $20 \%$ to the insulating gap shown in Fig. 9(d), and the remaining $80 \%$ is the impact of the Coulomb $U$.

We summarize the mechanisms responsible for the opening of the gap in Fig. 10 as follows. Altogether, we observe that the onset of the insulating gap in both tetragonal and monoclinic phases is due to the Hubbard $U$. On the mean-field level, this can only be realized if the $\pi_{g}$ orbitals around the Fermi level are split. While in the tetragonal case including a finite SOC is necessary to trigger the gap opening, the orbital degeneracy is broken in the monoclinic phase due to the lattice distortions.

\section{SUMMARY}

In this paper, we focus on two main questions: (i) what is the mechanism of symmetry breaking, responsible for the transition from the tetragonal to monoclinic phase, and (ii) how the crystal distortion influences the electronic properties of $\mathrm{KO}_{2}$.

Regarding the first problem, we performed the $a b$ initio 
studies of lattice dynamics for the tetragonal $14 / \mathrm{mmm}$ phase of $\mathrm{KO}_{2}$, in order to unveil the role of phonons in the structural transition. The lowest soft mode, found at the $\mathrm{N}$ point of the Brillouin zone, induces the monoclinic distortion. The obtained $C 2 / c$ symmetry and lattice parameters of the optimized structure agree with the diffraction data. Our study demonstrates a displacive character of the phase transition, in which the high- and lowtemperature phases are connected by the group-subgroup relation. In this transition, an important role is played by the coupling between the soft mode and electrons, which generates the splitting of molecular states $\pi_{g}$ on oxygen anions (Jahn-Teller effect). The monoclinic phase is stabilized by the local Coulomb interaction $U$, which allows for electron localization in the orbitally ordered state.

The implication of the structural transition on the electronic properties was investigated by the systematic studies of the band structures and electronic densities of states in both phases. In the tetragonal structure, the insulating state can be found by including simultaneously two on-site interactions: spin-orbit coupling and Hubbard $U$. In this case, a finite spin-orbit coupling removes the degeneracy, which triggers opening of the gap of the order of $0.5 \mathrm{eV}$. Interestingly, initiating an orbital order in the tetragonal phase also leads to a stable solution and to the gap of similar size - but also only once the local Hubbard interactions $\propto U$ are included. In the monoclinic phase, the orbital order and Mott insulator with the gap of the order of $1 \mathrm{eV}$ is naturally stabilized by the lattice distortion via the Jahn-Teller effect.

In conclusion, by performing the $\mathrm{DFT}+U$ studies of $\mathrm{KO}_{2}$, we revealed the displacive character of the structural transition from the high-temperature tetragonal to low-temperature monoclinic phase. We observe that the onset of the insulating gap in both tetragonal and monoclinic phase is due to the Hubbard $U$, provided that the orbital degeneracy at the Fermi level is removed.

\section{ACKNOWLEDGMENTS}

We thank Krzysztof Parlinski for valuable comments and discussions. This work was supported by the National Science Centre (NCN, Poland) under grants No.: 2017/25/B/ST3/02586 (O.S., D.G., A.P., M.S., and P.P.), 2016/22/E/ST3/00560 (K.W.), and 2016/23/B/ST3/00839 (D.G., K.W., and A.M.O.). A. M. Oleś is grateful for the Alexander von Humboldt Foundation Fellowship (Humboldt-Forschungspreis).

\section{Appendix A: Tetragonal structure with orbital order}

Here we discuss in detail the impact of the Coulomb interaction $U$ on the electronic structure in the tetragonal phase. In the calculations presented in Fig. 9 (left panels), even large values of the Coulomb $U$ lead to a metallic state unless the SOC is additionally included.

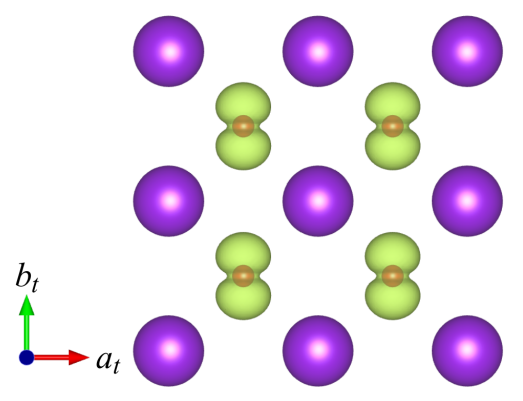

FIG. 11. Calculated isosurfaces of the hole density (in green) for the tetragonal phase with orbital order induced by enforced symmetry breaking. The image was rendered using VESTA software [32].

This can be understood as the result of tetragonal symmetry of the crystal structure (and thus charge density distribution) leading to the degeneracy of $\pi_{g}^{x}$ and $\pi_{g}^{y}$ orbitals. In the GGA $+U$ method, the gap cannot appear if the localized states around the Fermi level are degenerate.

It is possible however to obtain an insulating state by starting calculations from an orbitally ordered state and without symmetry constraint imposed on the wave function [52]. We can demonstrate this mechanism by first calculating the charge density for a cell with a small orthorhombic distortion leading to the ferro-orbital $\left(\pi_{g}^{y}\right)$ order shown in Fig. 11, and using it as an input for optimizing the tetragonal structure (without allowing the cell shape to change). Our results reveal that the orbitally ordered state has significantly lower energy $(0.2 \mathrm{eV} /$ f.u. $)$ than the state optimized without an orbital order, and a gap of $0.65 \mathrm{eV}$ opens in the electronic density of states as shown in Fig. 12(a). Including the SOC [Fig. 12(b)] does

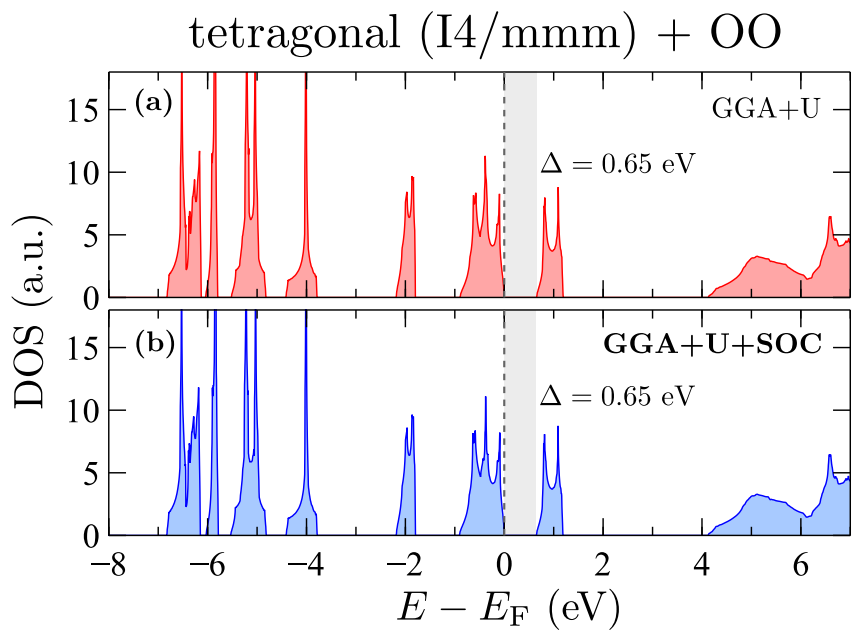

FIG. 12. Electronic density of states obtained for the tetragonal phase with initiated orbital order (unoccupied $\pi_{g}^{y}$ orbitals) within: (a) GGA $+U$ and (b) GGA $+U+\mathrm{SOC}$ calculations. 
(a)

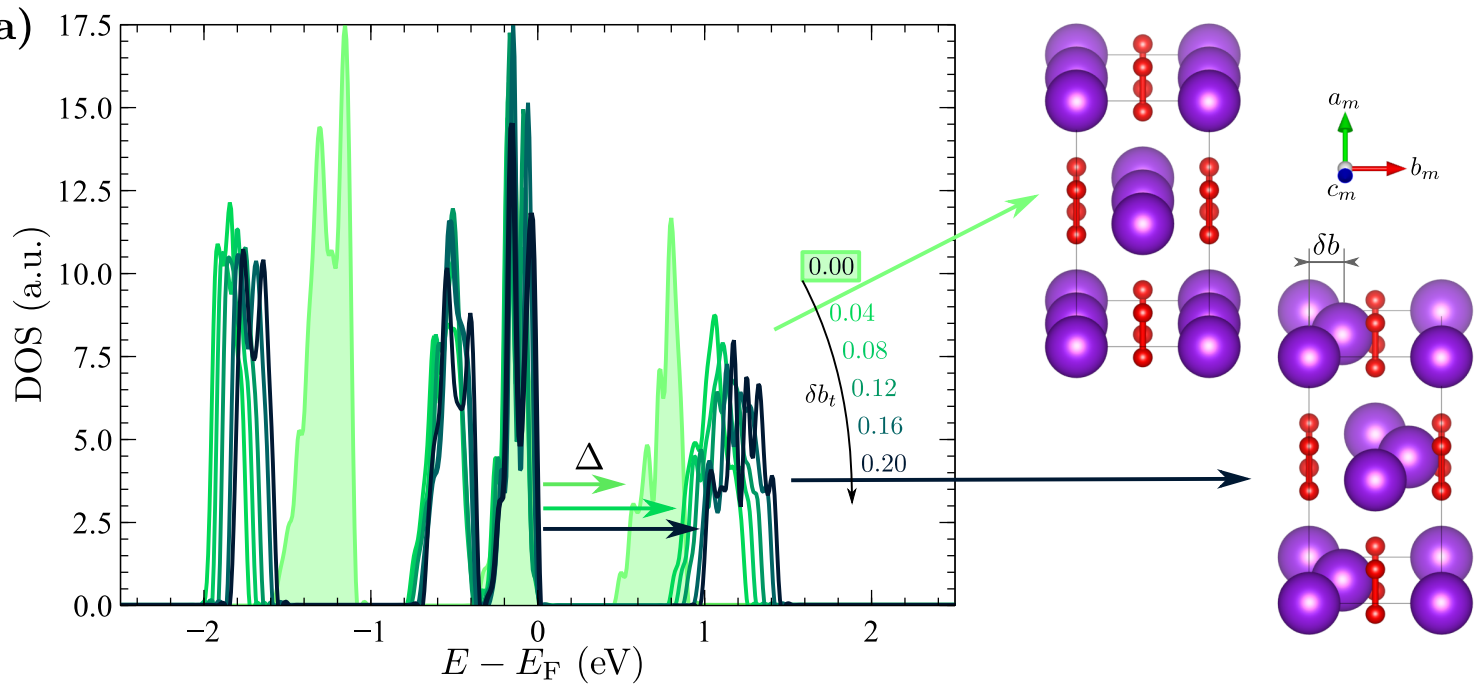

(b)

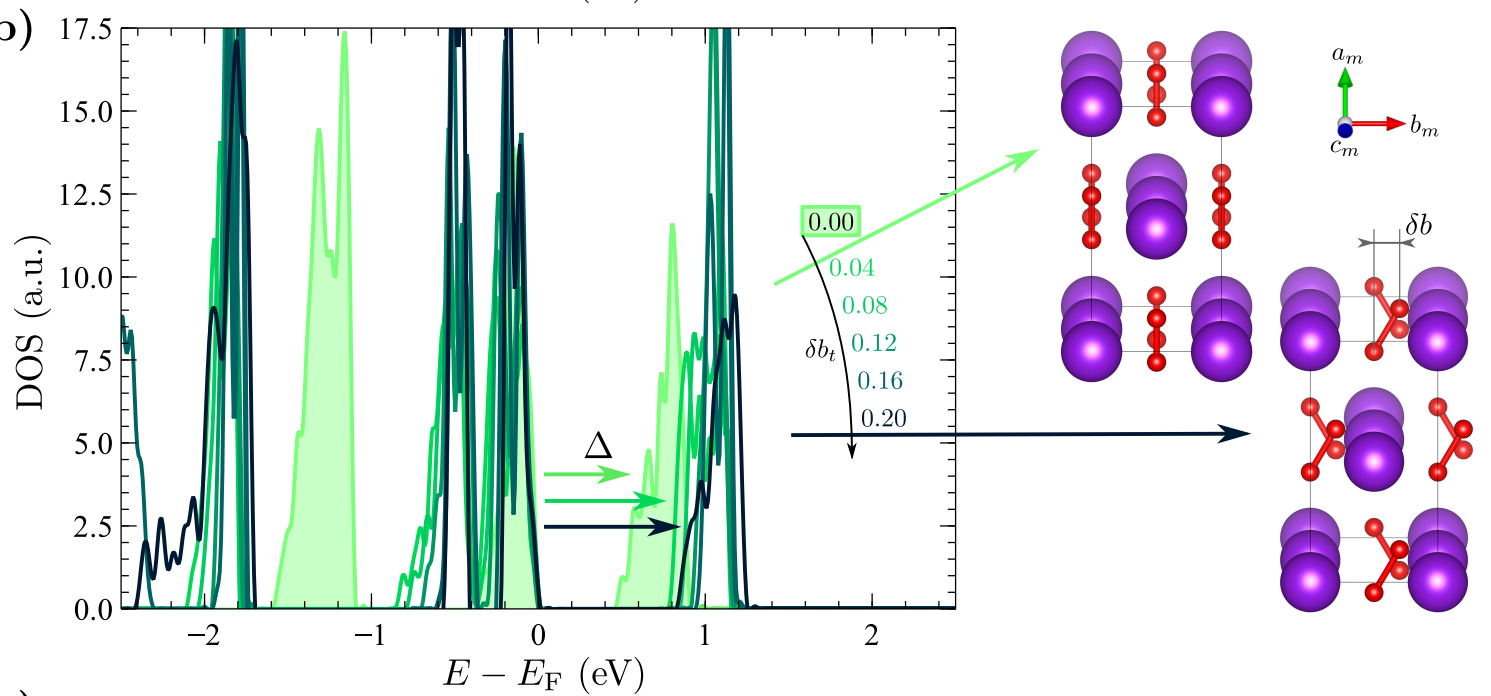

(c)

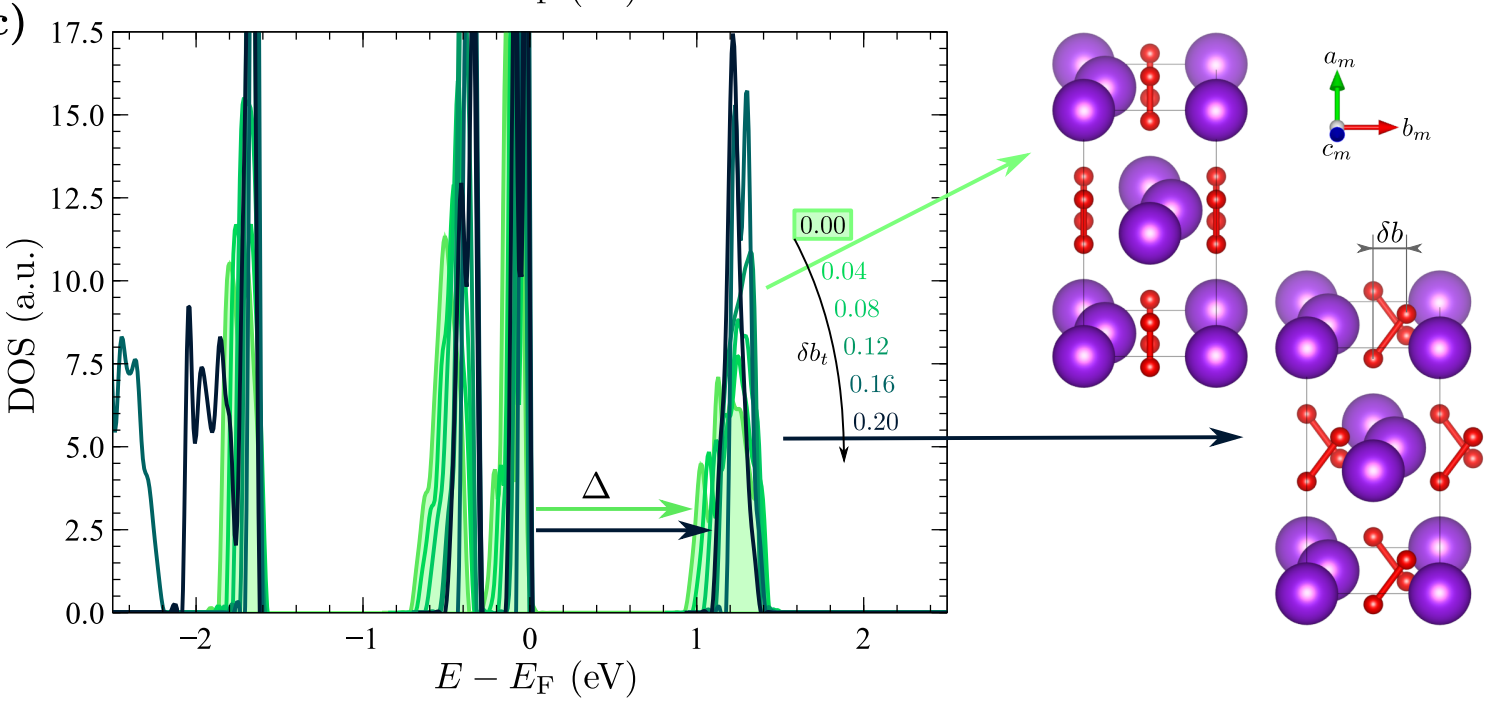

FIG. 13. Comparison of the electronic density of states (DOS) for different levels of partial crystal distortions in a simplified model of the monoclinic cell: (a) positions of $\mathrm{K}$ atoms are shifted without modification of $\mathrm{O}_{2}$ molecules; (b) oxygen molecules are tilted with $\mathrm{K}$ atoms in their tetragonal positions; (c) oxygen molecules are tilted in a lattice with potassium distortions already present. The image was rendered using VESTA software [32]. 
not induce any apparent changes to the electronic density of states, and this confirms that its role in opening of the gap presented in Fig. 9(e) is to split the states at the Fermi level. If the orbital order is present in the system, including the SOC does not influence significantly neither the electronic structure nor the total energy (similarly as in the monoclinic phase with the orbital order resulting from distortion).

Calculations for several different orbital orders in $\mathrm{RbO}_{2}$ [52] show that the lowering of total energy is similar in all cases (the relative differences are of the order of $10 \mathrm{meV}$ ). We note that a full relaxation of the system with orbital order breaking the tetragonal symmetry would always lead to a structural distortion.

\section{Appendix B: Impact of atomic displacement}

It is instructive to investigate the impact of atomic displacement on the insulating gap by evaluating the density of states (DOS) for a toy model of the monoclinic structure, in which we introduce separately artificial modifications of positions of $\mathrm{O}$ or $\mathrm{K}$ atoms, varying the size of the displacements. For this study we use a double tetragonal cell with lattice vectors chosen as in the monoclinic structure, and the simplified "monoclinic-like" modifications are illustrated in Fig. 13. This "toy-model" calculations, do not include e.g. different lattice vectors or volume of the true monoclinc cell.

Figure 13(a) demonstrates changes in the DOS upon shifting the potassium ions along the $b_{m}$ axis by distance $\delta b$, where $\delta b$ changes from 0.0 to 0.2 of $b_{m}$ (the last value roughly corresponds to the extent of the monoclinic dis- tortions). The results shown in light green with green background correspond to the tetragonal phase within the GGA $+U+$ SOC scheme [shown in Fig. 9(e) in the main part], with a small gap of about $0.5 \mathrm{eV}$. We observe that the smallest displacement with $\delta b=0.04 b_{m}$ causes already substantial changes: there is a jump in the gap size by about $0.35 \mathrm{eV}$ and a single peak below $-1 \mathrm{eV}$ separates into two. This can be understood by the different mechanism of the gap opening described in this work: even a small distortion from the tetragonal symmetry removes the degeneracy between $\pi_{g}$ orbitals, and the DOS is primarily determined by the cooperative effect of the resulting orbital order and the Coulomb $U$. The impact of the smallest $\delta b$ shown here provides another illustration of this mechanism. Further displacement modifies the electronic DOS only quantitatively, and the gap increases gradually to about $1 \mathrm{eV}$.

Figure 13(b) shows the analogue results for oxygen molecule rotations (with potassium ions in the tetragonal positions). Again, we observe a jump in the gap size after the smallest rotation, and then only minor changes (the gap varies between $0.8 \mathrm{eV}$ and $1 \mathrm{eV}$ ).

The last panel, Fig. 13(c), presents modifications of the electronic DOS upon oxygen molecule rotations, but starting from the structure with shifted potassium positions. As the orbital order and considerable distortions are present from the beginning, we observe only quantitative modifications of the DOS. It is worth noting, however, that only by simultaneous displacements of the potassium ions and rotations of oxygen molecules, we are able to obtain the gap of about $1.1 \mathrm{eV}$, i.e., similar to the result for the fully optimized monoclinic structure.
[1] Also called hyperoxides or dioxides.

[2] M. Hayyan, M. A. Hashim, and I. M. AlNashef, Superoxide ion: Generation and chemical implications, Chem. Rev. 116, 3029 (2016).

[3] M. Ziegler, H. R. Meister, and W. Känzig, Zur struktur von $\mathrm{KO}_{2}$ in der phase IV, Hel. Phys. Acta 48, 599 (1975).

[4] W. Känzig and M. Labhart, Molecular and magnetic order in alkali hyperoxides : A short review of recent work, J. Phys. Colloq. 37, C7 (1976).

[5] W. Kassatochkin and W. Kotow, Structure of potassium tetroxide, J. Chem. Phys. 4, 458 (1936).

[6] S. C. Abrahams and J. Kalnajs, The crystal structure of $\alpha$-potassium superoxide, Acta Cryst. 8, 503 (1955).

[7] A. Zumsteg, M. Ziegler, W. Känzig, and M. Bösch, Magnetische und kalorische eigenschaften von alkalihyperoxid-kristallen, in Physics of Condensed Matter, edited by G. Busch and S. Strässler (Springer Berlin Heidelberg, Berlin, Heidelberg, 1974) pp. 267-291.

[8] F. Astuti, M. Miyajima, T. Fukuda, M. Kodani, T. Nakano, T. Kambe, and I. Watanabe, Anionogenic magnetism combined with lattice symmetry in alkalimetal superoxide $\mathrm{RbO}_{2}$, J. Phys. Soc. Jpn. 88, 043701 (2019).
[9] S. Riyadi, B. Zhang, R. A. de Groot, A. Caretta, P. H. M. van Loosdrecht, T. T. M. Palstra, and G. R. Blake, Antiferromagnetic $S=1 / 2$ spin chain driven by $p$-orbital ordering in $\mathrm{CsO}_{2}$, Phys. Rev. Lett. 108, 217206 (2012).

[10] I. V. Solovyev, Spin-orbital superexchange physics emerging from interacting oxygen molecules in $\mathrm{KO}_{2}$, New J. Phys. 10, 013035 (2008).

[11] M. Kim, B. H. Kim, H. C. Choi, and B. I. Min, Antiferromagnetic and structural transitions in the superoxide $\mathrm{KO}_{2}$ from first principles: A $2 p$-electron system with spin-orbital-lattice coupling, Phys. Rev. B 81, 100409 (2010).

[12] K. Wohlfeld, M. Daghofer, and A. M. Oleś, Spinorbital physics for $p$ orbitals in alkali $\mathrm{RO}_{2}$ hyperoxidesgeneralization of the Goodenough-Kanamori rules, EPL (Europhysics Letters) 96, 27001 (2011).

[13] G. F. Carter, J. L. Margrave, and D. H. Templeton, A high-temperature crystal modification of $\mathrm{KO}_{2}$, Acta Cryst. 5, 851 (1952).

[14] H. G. Smith, R. M. Nicklow, L. J. Raubenheimer, and M. K. Wilkinson, Antiferromagnetism in potassium superoxide $\mathrm{KO}_{2}$, J. Appl. Phys. 37, 1047 (1966).

[15] M. Labhart, D. Raoux, W. Känzig, and M. A. Bösch, 
Magnetic order in $2 p$-electron systems: Electron paramagnetic resonance and antiferromagnetic resonance in the alkali hyperoxides $\mathrm{KO}_{2}, \mathrm{RbO}_{2}$, and $\mathrm{CsO}_{2}$, Phys. Rev. B 20, 53 (1979).

[16] M. E. Lines and M. A. Bösch, Magnetogyration, Phys. Rev. B 23, 263 (1981).

[17] F. Halverson, Comments on potassium superoxide structure, J. Phys. Chem. Solids 23, 207 (1962).

[18] A. U. Khan and S. D. Mahanti, Collective electron effects of $\mathrm{O}_{2}^{-}$in potassium superoxide, J. Chem. Phys. 63, 2271 (1975).

[19] J.-S. Kang, D. H. Kim, J. H. Hwang, J. Baik, H. J. Shin, M. Kim, Y. H. Jeong, and B. I. Min, Soft x-ray absorption and photoemission spectroscopy study of superoxide $\mathrm{KO}_{2}$, Phys. Rev. B 82, 193102 (2010).

[20] N. R. Mathiesen, S. Yang, J. M. García-Lastra, T. Vegge, and D. J. Siegel, Charge transport in alkali-metal superoxides: A systematic first-principles study, Chem. Mater. 31, 9156 (2019).

[21] A. K. Nandy, P. Mahadevan, P. Sen, and D. D. Sarma, $\mathrm{KO}_{2}$ : Realization of orbital ordering in a $p$-orbital system, Phys. Rev. Lett. 105, 056403 (2010).

[22] A. K. Nandy, P. Mahadevan, and D. D. Sarma, $\mathrm{K}_{2} \mathrm{O}_{2}$ : The most stable oxide of K, Phys. Rev. B 84, 035116 (2011).

[23] A. K. Nandy, P. Mahadevan, and D. D. Sarma, Formation energies and the stability of the oxides of K, Molecular Simulation 38, 1308 (2012).

[24] M. Kim and B. I. Min, Temperature-dependent orbital physics in a spin-orbital-lattice-coupled $2 p$ electron Mott system: The case of $\mathrm{KO}_{2}$, Phys. Rev. B 89, 121106 (2014).

[25] P. E. Blöchl, Projector augmented-wave method, Phys. Rev. B 50, 17953 (1994).

[26] G. Kresse and J. Hafner, Ab initio molecular-dynamics simulation of the liquid-metal-amorphous-semiconductor transition in germanium, Phys. Rev. B 49, 14251 (1994).

[27] G. Kresse and J. Furthmüller, Efficient iterative schemes for ab initio total-energy calculations using a plane-wave basis set, Phys. Rev. B 54, 11169 (1996).

[28] G. Kresse and D. Joubert, From ultrasoft pseudopotentials to the projector augmented-wave method, Phys. Rev. B 59, 1758 (1999).

[29] J. P. Perdew, K. Burke, and M. Ernzerhof, Generalized gradient approximation made simple, Phys. Rev. Lett. 77, 3865 (1996).

[30] A. I. Liechtenstein, V. I. Anisimov, and J. Zaanen, Density-functional theory and strong interactions: Orbital ordering in Mott-Hubbard insulators, Phys. Rev. B 52, R5467 (1995).

[31] S. Steiner, S. Khmelevskyi, M. Marsmann, and G. Kresse, Calculation of the magnetic anisotropy with projected-augmented-wave methodology and the case study of disordered $\mathrm{Fe}_{1-x} \mathrm{Co}_{x}$ alloys, Phys. Rev. B 93, 224425 (2016).

[32] K. Momma and F. Izumi, vesta3 for three-dimensional visualization of crystal, volumetric and morphology data, J. Appl. Crystallogr. 44, 1272 (2011).

[33] H. J. Monkhorst and J. D. Pack, Special points for Brillouin-zone integrations, Phys. Rev. B 13, 5188 (1976).

[34] K. Parlinski, Z. Q. Li, and Y. Kawazoe, First-principles determination of the soft mode in cubic $\mathrm{ZrO}_{2}$, Phys. Rev. Lett. 78, 4063 (1997).
[35] K. Parlinski, Phonon, Cracow (2018).

[36] M. Gajdoš, K. Hummer, G. Kresse, J. Furthmüller, and F. Bechstedt, Linear optical properties in the projectoraugmented wave methodology, Phys. Rev. B 73, 045112 (2006).

[37] W. Hesse, M. Jansen, and W. Schnick, Recent results in solid state chemistry of ionic ozonides, hyperoxides, and peroxides, Progress in Solid State Chemistry 19, 47 (1989).

[38] P. D. C. Dietzel, R. K. Kremer, and M. Jansen, Tetraorganylammonium superoxide compounds: Close to unperturbed superoxide ions in the solid state, Journal of the American Chemical Society 126, 4689 (2004).

[39] M. Pedio, Z. Y. Wu, M. Benfatto, A. Mascaraque, E. Michel, C. Ottaviani, C. Crotti, M. Peloi, M. Zacchigna, and C. Comicioli, NEXAFS experiment and multiple scattering calculations on $\mathrm{KO}_{2}$ : Effects on the $\pi$ resonance in the solid phase, Phys. Rev. B 66, 144109 (2002).

[40] H. Seyeda and M. Jansen, A novel access to ionic superoxides and the first accurate determination of the bond distance in $\mathrm{O}_{2}^{-}$, J. Chem. Soc., Dalton Trans. , 875 (1998).

[41] J. Bates, M. Brooker, and G. Boyd, Raman spectra of $\mathrm{O}^{-2}$ and $\mathrm{O}^{-3}$ ions in alkali-metal superoxides and ozonides, Chem. Phys. Lett. 16, 391 (1972).

[42] Y. Wang, S. Wang, Y. Zhang, J. Lv, Y. Chen, W. Zheng, and Y. Ma, Ground-state crystal structure of strontium peroxide predicted from first principles, Inorg. Chem. 56, 7545 (2017).

[43] V. Jovic, R. J. Koch, S. K. Panda, H. Berger, P. Bugnon, A. Magrez, K. E. Smith, S. Biermann, C. Jozwiak, A. Bostwick, E. Rotenberg, and S. Moser, Dirac nodal lines and flat-band surface state in the functional oxide $\mathrm{RuO}_{2}$, Phys. Rev. B 98, 241101 (2018).

[44] J. M. Kahk, C. G. Poll, F. E. Oropeza, J. M. Ablett, D. Céolin, J.-P. Rueff, S. Agrestini, Y. Utsumi, K. D. Tsuei, Y. F. Liao, F. Borgatti, G. Panaccione, A. Regoutz, R. G. Egdell, B. J. Morgan, D. O. Scanlon, and D. J. Payne, Understanding the electronic structure of $\mathrm{IrO}_{2}$ using hard-X-ray photoelectron spectroscopy and density-functional theory, Phys. Rev. Lett. 112, 117601 (2014).

[45] Y. Sun, Y. Zhang, C.-X. Liu, C. Felser, and B. Yan, Dirac nodal lines and induced spin Hall effect in metallic rutile oxides, Phys. Rev. B 95, 235104 (2017).

[46] P. K. Das, J. Sławińska, I. Vobornik, J. Fujii, A. Regoutz, J. M. Kahk, D. O. Scanlon, B. J. Morgan, C. McGuinness, E. Plekhanov, D. Di Sante, Y.-S. Huang, R.-S. Chen, G. Rossi, S. Picozzi, W. R. Branford, G. Panaccione, and D. J. Payne, Role of spin-orbit coupling in the electronic structure of $\mathrm{IrO}_{2}$, Phys. Rev. Materials 2, 065001 (2018).

[47] X. Xu, J. Jiang, W. J. Shi, V. Süß, C. Shekhar, S. C. Sun, Y. J. Chen, S.-K. Mo, C. Felser, B. H. Yan, H. F. Yang, Z. K. Liu, Y. Sun, L. X. Yang, and Y. L. Chen, Strong spin-orbit coupling and Dirac nodal lines in the three-dimensional electronic structure of metallic rutile $\mathrm{IrO}_{2}$, Phys. Rev. B 99, 195106 (2019).

[48] F. Herman, C. D. Kuglin, K. F. Cuff, and R. L. Kortum, Relativistic corrections to the band structure of tetrahedrally bonded semiconductors, Phys. Rev. Lett. 11, 541 (1963).

[49] K. V. Shanavas, Z. S. Popović, and S. Satpathy, Theoretical model for Rashba spin-orbit interaction in $d$ electrons, 
Phys. Rev. B 90, 165108 (2014).

[50] The $A$-type AFM ordering means ferromagnetic planes which are stacked antiferromagnetically, cf. the $A$-AFM order discussed in the context of e.g.: $\mathrm{LaMnO}_{3}$ [55], $\mathrm{GdFeO}_{3}[56]$ or the several double perovskites [57]. Note that in Ref. [12] the same type of magnetic order as discussed here was referred to as $C$-type AFM, for in that paper the types of magnetic order were defined by the ratio of the ferromagnetic to the antiferromagnetic bonds.

[51] A. Avella, A. M. Oleś, and P. Horsch, Defect-induced orbital polarization and collapse of orbital order in doped vanadium perovskites, Phys. Rev. Lett. 122, 127206 (2019).

[52] R. Kováčik and C. Ederer, Correlation effects in $p$ electron magnets: Electronic structure of $\mathrm{RbO}_{2}$ from first principles, Phys. Rev. B 80, 140411 (2009).

[53] R. Kováčik, P. Werner, K. Dymkowski, and C. Ederer, Rubidium superoxide: A p-electron Mott insulator, Phys.
Rev. B 86, 075130 (2012).

[54] For $U=0$, the deviation of $\mathrm{O}_{2}^{-}$molecules from the tetragonal $c_{t}$ axis as well as potassium ions displacements are substantially reduced. Therefore, in order to investigate the impact of the full monoclinic distortion, we use the unrelaxed geometry.

[55] E. O. Wollan and W. C. Koehler, Neutron diffraction study of the magnetic properties of the series of perovskite-type compounds $[(1-\mathrm{x}) \mathrm{La}, \mathrm{xCa}] \mathrm{MnO}_{3}$, Phys. Rev. 100, 545 (1955).

[56] X.-H. Zhu, X.-B. Xiao, X.-R. Chen, and B.-G. Liu, Electronic structure, magnetism and optical properties of orthorhombic GdFeO3 from first principles, RSC Adv. 7, 4054 (2017).

[57] Y. Aharbil, H. Labrim, S. Benmokhtar, M. A. Haddouch, L. Bahmad, and L. Laanab, Self-consistent (DFT+U) study of electronic, structural and magnetic properties in $\mathrm{A}_{2} \mathrm{NiMoO}_{6}(\mathrm{~A}=\mathrm{Ba}, \mathrm{Sr})$ compounds, Mater. Res. Express. 3, 086104 (2016). 